\title{
A STUDY OF FEMORAL TORSION IN CHILDREN
}

\author{
Anthony McSweeny, Brisbane, Australia
}

Techniques for measuring upper femoral torsion in children have been progressively developed by Dunn (1952), Ryder and Crane (1953), Dunlap, Shands, Hollister, Gaul and Streit (1953), Magilligan (1956) and Shands and Steele (1958). The projected neck-shaft angle of inclination and the projected angle of femoral torsion (Fig. 1) may be used to plot the true torsion on a table based on a mathematically developed geometrical relationship (Dunn 1952, Ryder and Crane 1953, Dunlap et al. (1953), Magilligan 1956, Shands and Steele 1958). The essential features of the radiographic positioning include an antero-posterior view for inclination taken in neutral rotation and a view for torsion with hip and knee each flexed to a right angle and with the hip abducted to a fixed and known angle between 10 degrees and 30 degrees. For this study the technique of Ryder and Crane (1953) has been used, and for normal values the figures of Shands and Steele (1958) which vary little from those of Ryder and Crane (1953) and Crane (1959) have been averaged with the latter (Table I). Only figures at least 10 degrees over or under the normal were recorded as abnormal torsion.

In the past ten years 1,320 children presenting consecutively were examined personally to determine the incidence and sequelae of toeing-in and toeing-out when walking. In the

TABLE I

Normal Values for ANTEVersion

\begin{tabular}{|cccc|}
\hline $\begin{array}{c}\text { Age } \\
\text { (years) }\end{array}$ & $\begin{array}{c}\text { Anteversion (degrees) } \\
\text { Dunlap et al., } \\
\text { Shands and Steele }\end{array}$ & $\begin{array}{c}\text { Ryder and Crane } \\
\text { (1953) }\end{array}$ & Average \\
\hline $1^{\frac{1}{2}}$ & 39 & 41 & 40 \\
2 & 35 & 37 & 36 \\
3 & 31 & 37 & 34 \\
4 & 30 & 35 & 32 \\
5 & 29 & 26 & 30 \\
6 & 28 & 26 & 26 \\
7 & 27 & 25 & 25 \\
8 & 26 & 24 & 23 \\
9 & 25 & & \\
10 & 25 & & \\
11 & 24 & & \\
12 & 24 & & \\
13 & 24 & & \\
14 & 23 & & \\
15 & 22 & & \\
16 & 18 & & \\
over 16 & 16 & & \\
\hline
\end{tabular}

main these children were presented for advice on minor postural defects and injuries to bone and joint. Children with known causes of anteversion such as congenital dislocation of the hip, Legg-Perthes' disease, cerebral palsy and congenital talipes equinovarus were excluded from this series. The children studied thus represented a near normal sample of the community.

Of these children, 180 (13.6 per cent) showed toeing-in (174 cases) and toeing-out (six cases), confirmed by significant alteration in the range of rotation of the hip as measured with the child lying prone with knees flexed to a right angle so that the lower leg indicated the 

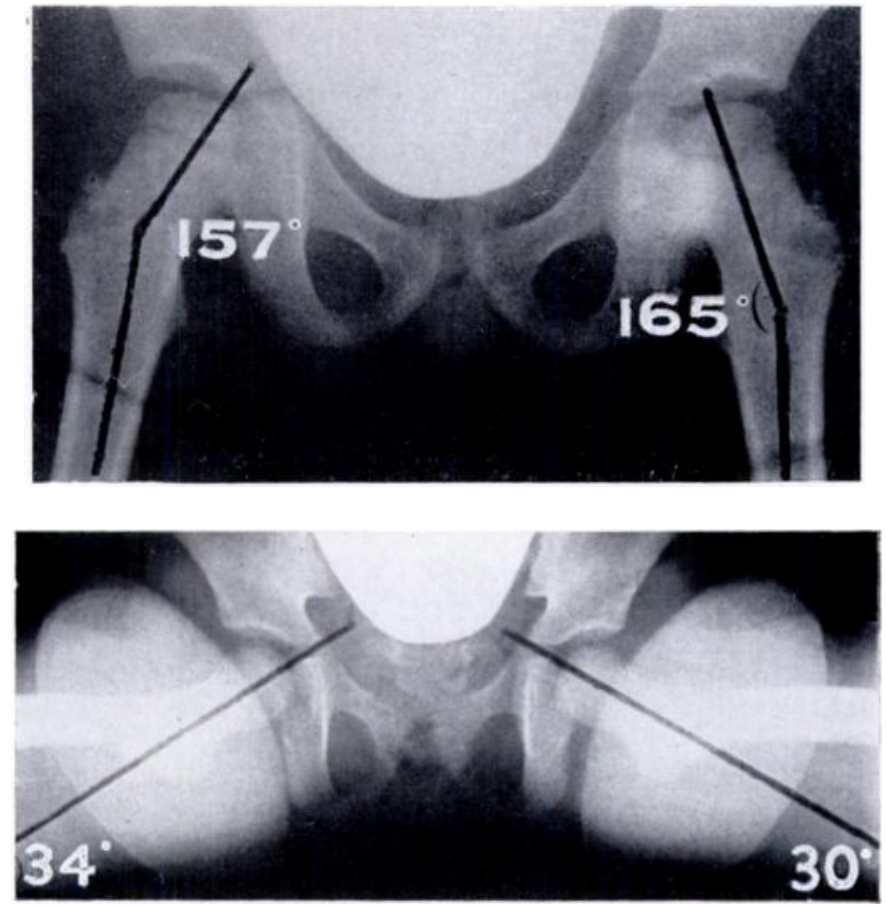

FIG. 1

Radiographs showing method of measurement of neck-shaft angle and angle of torsion.

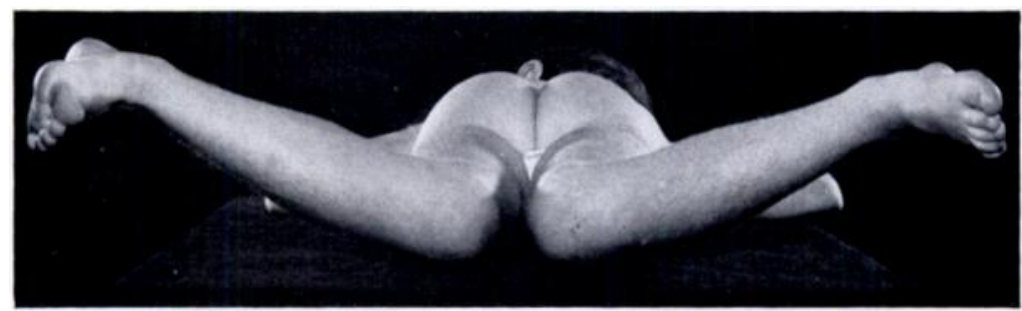

FIG. 2

Abnormally large range of medial rotation.

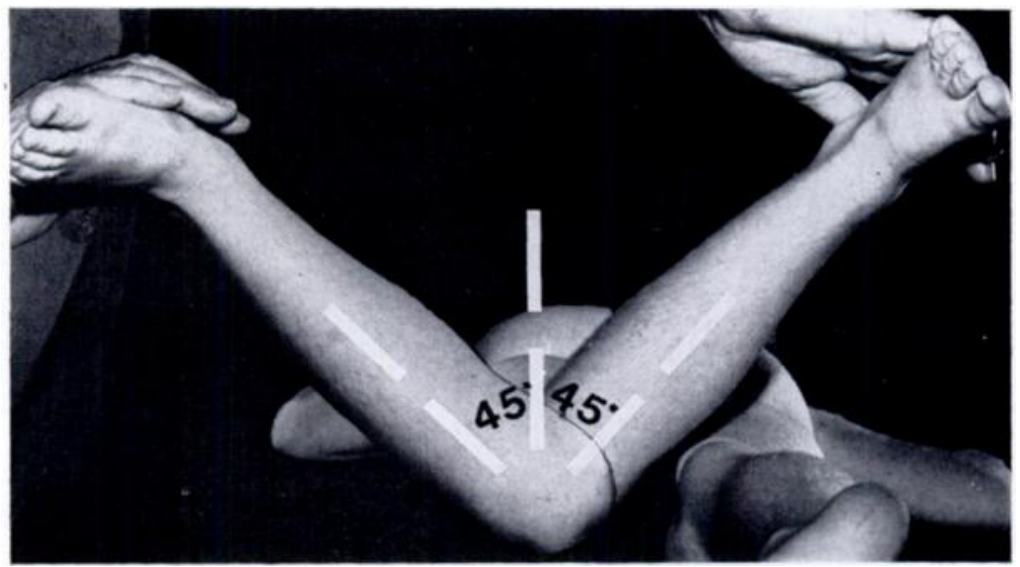

Fig. 3

Normal range of medial and lateral rotation.

VOL. 53 B, NO. 1, FEBRUARY 1971 
angle of rotation (Fig. 2). Care was taken to hold the pelvis firmly on the table while passively moving the lower leg through the permitted arc (Fig. 3); 45 degrees of movement on either side of the vertical was regarded as normal.

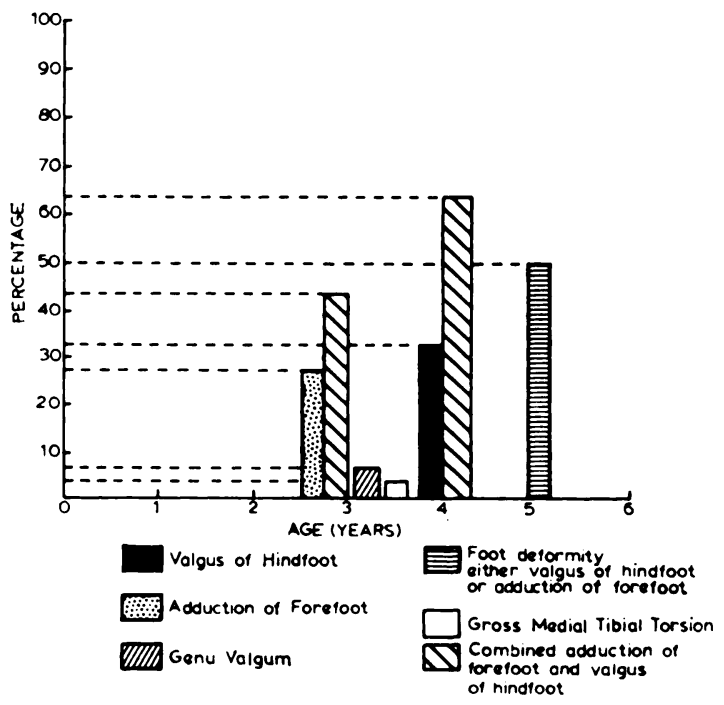

FIG. 4

Associated deformities in 174 children showing toeing-in.

In the 174 cases of toeing-in there were associated defects as follows (Fig. 4). 1) Adduction of the forefoot in 25 per cent and a combined adduction of the forefoot and hindfoot valgus

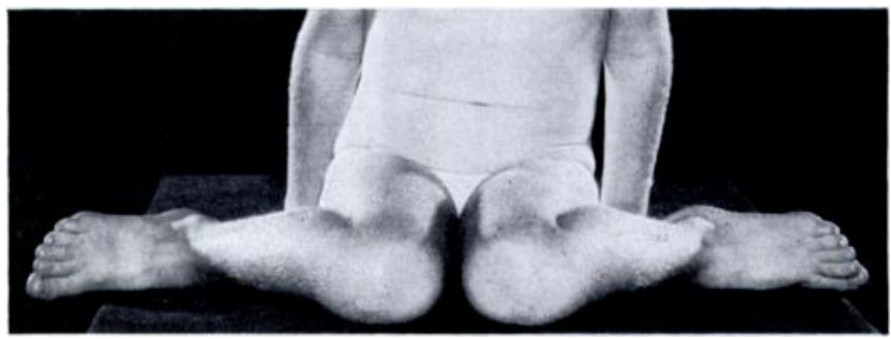

Fig. 5

Persistent "frog-sitting" habit.

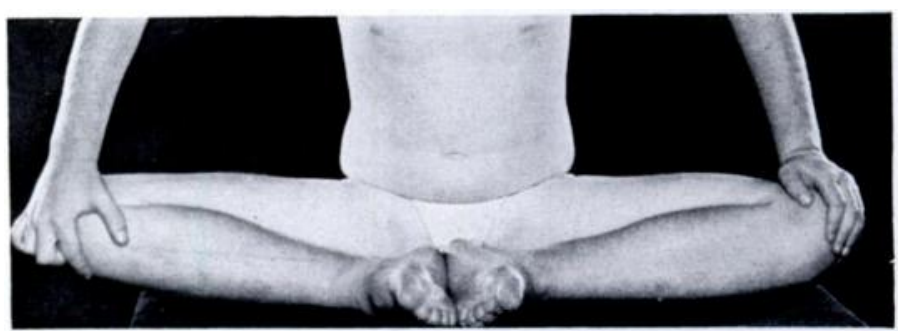

FIG. 6

Assisted "tailor-sitting" treatment.

in 44 per cent at an average age of $2 \cdot 7$ years. 2) Valgus of the hindfoot in 33 per cent and a combined adduction of the forefoot and hindfoot valgus in 65 per cent at an average age of four years. 3) Foot deformity in 50 per cent, either adduction of the forefoot or hindfoot 
valgus, at the average age of correction of toeing-in, five years. 4) Genu valgum with more than 8.7 centimetres between the medial malleoli on standing in 8.3 per cent at an average age of 3.3 years. 5) Gross medial tibial torsion in 3.3 per cent at an average age of 3.5 years. 6) Persistent frog-sitting habit in uncorrected intoeing of about one-third (Fig. 5). 7) Restricted lateral rotation complained of by ballet teachers in six subjects, two of whom had pain in the hips and one of whom had knee pain. Sixty-two patients were followed beyond the age of expected correction (five years) to correlate the clinical progress with the radiological.

Of the six cases of toeing-out, four corrected between the ages of two and four and a half, and three of these, who earlier demonstrated torsion greater than minus 10 degrees, had normal torsion when toeing-out was normal. The constant wearing of Thomas heels was their only treatment.

The treatment for toeing-in comprised assisted tailor-sitting for all (Fig. 6) and corrected footwear which included Thomas heels for hindfoot valgus and reversed boots for adduction of the forefoot. A combination of both corrections on the boots was used when both deformities were present. Derotation femoral osteotomies were performed in one case.

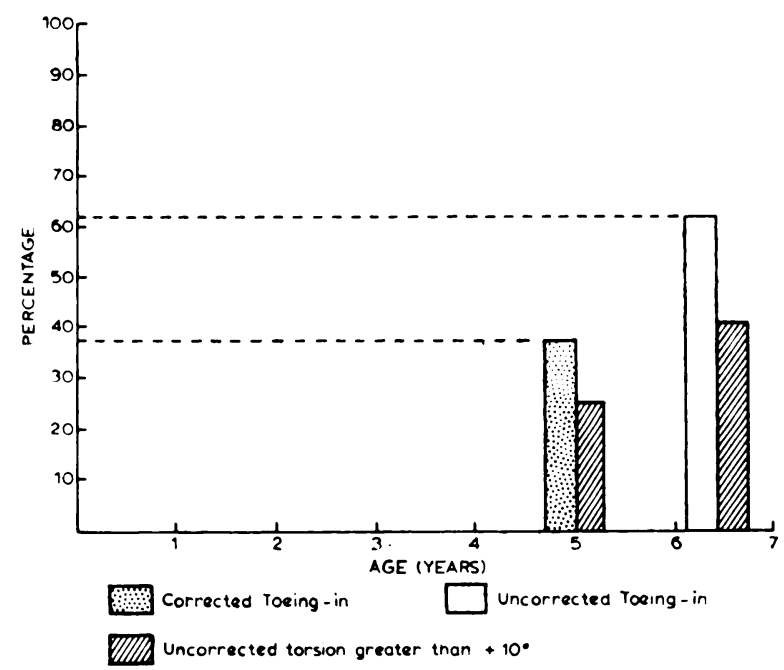

Fig. 7

Progress of toeing-in in fifty-six children followed beyond 5 years of age.

Of the fifty-six patients with toeing-in, twenty-two (39 per cent) had ceased to do so at an average age of five years; of those presenting between one and a half to two and a half years the average age of correction was four years, while those presenting between four and seven years reverted to normal at an average age of seven years (Fig. 7). Torsion of not less than 10 degrees persisted in approximately two-thirds of patients who had ceased to toe-in. Thus, only about one-third of patients in whom toeing-in had ceased had normal angles of anteversion. Thirtyfour children (61 per cent) had not reverted to normal at an average age of 6.4 years and of these, approximately two-thirds showed persistent radiological torsion of at least 10 degrees. These findings are comparable to those of Harris (1965). The remaining one-third had normal angles of anteversion.

There were 123 girls and fifty-seven boys in the whole group-a ratio of about seven to three. This ratio decreased to four to three in patients who did not undergo spontaneous correction. The more satisfactory response of the girls could be related to joint laxity caused by hormonal influences in the female (Wilkinson 1963). 


\section{DISCUSSION}

The 13.6 per cent incidence of toeing-in or toeing-out in the 1,320 near normal subjects suggests a significant incidence of persistent foetal alignment (Somerville 1953, 1957). That two-thirds of corrected or uncorrected toeing-in was associated with persistent upper femoral torsion of at least 10 degrees above normal at an average age of five years suggests continuing stress on the hips, knees and feet, with attempted normal toeing-out gait perhaps accounting for the unexplained "growing pains" of childhood. Although there was no means of assessing stress at the hips there was a one-third incidence of valgus of the hindfoot at average age of four years. This was interpreted as a compensatory foot deformity to accommodate for uncorrected torsion. A two-thirds incidence of combined hindfoot valgus and forefoot adduction at four years is also interpreted as compensatory hindfoot valgus for forefoot adduction, which itself may be secondary to anteversion.

On these findings, which indicate the poor results of conservative treatment, Somerville's (1963) contention that derotational osteotomy is justified before secondary deformities occur bears consideration, at least in cases of marked persisting torsion.

The fact that one-third of the patients whose intoeing was not corrected showed no abnormal torsion as measured radiologically at an average of 6.4 years suggests an abnormal anterior acetabular rotation demanding intoeing. Because these patients have persistent foetal alignment (of acetabular origin) their hips are subjected to the same stresses as those of newborn infants. According to Salter (1966) their hips when extended or adducted tend to be forced out of the acetabulum. Salter, Kostuik and Schatzker (1963) showed that in newborn pigs with one hip maintained in extension for eight weeks and the other flexed, acetabular maldirection occurred in the extended hip only.

Prevention of torsional stresses is probably worth while in the early years of life by parental education to avoid prone lying and frog sitting, nowadays often habitual postures in front of television, at least in Australia. As McKibbin (1968) has shown, the psoas is shortened in the frog position and sitting repeatedly in this manner may be expected to aggravate any tendency to anteversion.

Persistent upper femoral torsion in two-thirds of all patients with toeing-in, whether corrected or uncorrected, suggests the presence of abnormal stresses at hips, knees and feet which could later be significant in the etiology of osteoarthritis at these levels of stress. If to this is added the strong evidence of maldirection of the acetabulum in one-third of children with uncorrected toeing-in who have normal upper femoral torsion, then it is reasonable to speculate on rotational stresses persisting into middle age and predisposing to osteoarthritis.

\section{SUMMARY}

1. After exclusion of the well known causes of torsion such as congenital dislocation of the hip, Legg-Perthes' disease, cerebral palsy and congenital talipes equinovarus, examination of 1,320 nearly normal children disclosed an incidence of 13.6 per cent toeing-in or toeing-out.

2. There was a female preponderance of seven to three on presentation decreasing to four to three in uncorrected cases.

3. There was persisting upper femoral torsion of greater than 10 degrees in two-thirds of all cases of toeing-in whether corrected or uncorrected.

4. When correction of toeing-in occurred it took place between four and seven years of age, but mainly at five years.

5. There was a normal angle of anteversion in one-third of children whose toeing-in was not corrected. This suggests the possibility of acetabular maldirection.

6. Uncorrected torsional stresses may play a part in the later development of osteoarthritis. 
REFERENCES

Crane, L. (1959): Femoral Torsion and Its Relation to Toeing-in and Toeing-out. Journal of Bone and Joint Surgery, 41-A, 421.

Dunlap, K., Shands, A. R., Jun., Hollister, L. C., Jun., Gaul, J. S., Jun., and Streit, H. A. (1953): A New Method for Determination of Torsion of the Femur. Journal of Bone and Joint Surgery, 35-A, 289.

Dunn, D. M. (1952): Anteversion of the Neck of the Femur. Journal of Bone and Joint Surgery, 34-B, 181.

HARRIS, N. H. (1965): A Method of Measurement of Femoral Neck Anteversion and a Preliminary Report on its Practical Application. Journal of Bone and Joint Surgery, 47-B, 188.

McKibBin, B. (1968): The Action of the Iliopsoas Muscle in the Newborn. Journal of Bone and Joint Surgery, 50-B, 161.

Magilligan, D. J. (1956): Calculation of the Angle of Anteversion by Means of Horizontal Lateral Roentgenography. Journal of Bone and Joint Surgery, 38-A, 1231.

Ryder, C. T., and Crane, L. (1953): Measuring Femoral Anteversion: The Problem and a Method. Journal of Bone and Joint Surgery, 35-A, 321.

Salter, R. B. (1966): The Present State of Innominate Osteotomy in Congenital Dislocation of the Hip. Journal of Bone and Joint Surgery, 48-B, 853.

Salter, R. B., Kostuik, J., and Schatzker, J. (1963): Experimental Dysplasia of the Hip and Its Reversibility in Newborn Pigs. Journal of Bone and Joint Surgery, 45-A, 1781.

Shands, A. R., Jun., and Steele, M. K. (1958): Torsion of the Femur. Journal of Bone and Joint Surgery, 40-A, 803.

Somerville, E. W. (1953): Development of Congenital Dislocation of the Hip. Journal of Bone and Joint Surgery, 35-B, 568.

Somerville, E. W. (1957): Persistent Foetal Alignment. Journal of Bone and Joint Surgery, 39-B, 106.

Somerville, E. W. (1963): Rotational Abnormalities of the Lower Limb. Journal of Bone and Joint Surgery, 45-B, 622.

Wilkinson, J. A. (1963): Prime Factors in the Etiology of Congenital Dislocation of the Hip. Journal of Bone and Joint Surgery, 45-B, 268.

VOL. 53 B, NO. 1, FEBRUARY 1971 